\title{
Connexin 26 regulates epidermal barrier and wound remodeling and promotes psoriasiform response
}

\author{
Ali R. Djalilian, ${ }^{1,2}$ David McGaughey, ${ }^{1,2}$ Satyakam Patel, ${ }^{1}$ Eun Young Seo, ${ }^{1}$ Chenghua Yang, ${ }^{1}$
} Jun Cheng, ${ }^{1}$ Melanija Tomic, ${ }^{3}$ Satrajit Sinha, ${ }^{4}$ Akemi Ishida-Yamamoto, ${ }^{5}$ and Julia A. Segre ${ }^{1}$

${ }^{1}$ National Human Genome Research Institute, ${ }^{2}$ National Eye Institute, and ${ }^{3}$ National Institute of Child Health and Development, NIH, Bethesda, Maryland, USA ${ }^{4}$ State University of New York at Buffalo, Buffalo, New York, USA. ${ }^{5}$ Asahikawa Medical College, Asahikawa, Japan.

\begin{abstract}
Inflammatory skin disorders result in significant epidermal changes, including keratinocyte hyperproliferation, incomplete differentiation, and impaired barrier. Here we test whether, conversely, an impaired epidermal barrier can promote an inflammatory response. Mice lacking the transcription factor Kruppel-like factor 4 (Klf4) have a severe defect in epidermal barrier acquisition. Transcription profiling of $\mathrm{Klf4}^{-/-}$newborn skin revealed similar changes in gene expression to involved psoriatic plaques, including a significant upregulation of the gap junction protein connexin 26 (Cx26). Ectopic expression of $\mathrm{Cx} 26$ from the epidermis-specific involucrin (INV) promoter (INV-Cx26) demonstrated that downregulation of $\mathrm{Cx} 26$ is required for barrier acquisition during development. In juvenile and adult mice, persistent $\mathrm{Cx} 26$ expression kept wounded epidermis in a hyperproliferative state, blocked the transition to remodeling, and led to an infiltration of immune cells. Mechanistically, ectopic expression of $\mathrm{Cx} 26$ in keratinocytes resulted in increased ATP release, which delayed epidermal barrier recovery and promoted an inflammatory response in resident immune cells. These results provide a molecular link between barrier acquisition in utero and epidermal remodeling after wounding. More generally, these studies suggest that the most effective treatments for inflammatory skin disorders might concomitantly suppress the immune response and enhance epidermal differentiation to restore the barrier.
\end{abstract}

\section{Introduction}

The epidermis serves as a barrier between the complex physiology of the body and an often hostile environment $(1,2)$. Many medical problems and diseases are caused or aggravated by defects of the barrier. Premature infants are at increased risk for infection and dehydration because of incomplete development of the epidermal barrier (3). Proper wound healing recapitulates many of these epidermal developmental processes, including reestablishment of the barrier. Trauma to the epidermis, resulting in both a breach in the epidermal barrier and immune cell activation, is a common trigger of an outbreak of psoriasis, a chronic inflammatory skin disorder with a prevalence of 2-3\% in Europe and North America $(4,5)$. Trauma-initiated psoriatic lesions, known as the isomorphic phenomenon, underscores the complex relationship between the keratinocytes and the other cell populations resident in the skin. The pathogenesis of psoriasis is complex, with genetic and environmental factors affecting both focal activation of Tlymphocytes and aberrant proliferation and differentiation of keratinocytes. Much evidence, including novel $\mathrm{T}$ cell-directed therapies, support a T lymphocyte-based pathogenesis for psoriasis. However, strong evidence for an epidermal contribution comes from linkage of psoriasis susceptibility to the epidermal differentiation complex, a locus of approximately 50 genes encoding proteins that regulate and build the epidermal barrier. These genetic data suggest that either impaired barrier or barrier recovery may contribute to pso-

Nonstandard abbreviations used: Cx26, connexin 26; K, keratin; Klf4, Kruppel-like factor 4; Inv, involucrin; PPADS, pyridoxalphosphate-6-azophenyl-2',4'-disulfonic acid; SPRR, small proline-rich; TSLP, thymic stromal lymphopoietin.

Conflict of interest: The authors have declared that no conflict of interest exists. Citation for this article: J. Clin. Invest. 116:1243-1253 (2006). doi:10.1172/JCI27186. riatic progression (6). The extent of the barrier dysfunction correlates with the degree of inflammation within the psoriatic lesion (7). Barrier disruption stimulates both keratinocyte proliferation and cytokine production, features of skin inflammatory diseases $(8,9)$. Interactions between immune and epidermal cells raise the possibility that a positive feedback loop between impaired barrier and inflammatory response exists. Here we examine the molecular similarities between the terminal stages of epidermal development and wound healing, as well as elements of psoriatic progression.

We have shown previously that the transcription factor Kruppellike factor 4 (Klf4) is necessary to establish the murine epidermal barrier in utero (10). The epidermis acquires the ability to function as a permeability barrier in anticipation of the transition to the terrestrial environment at E18 of a 19-day gestation period for mice (11). The transcription factor Klf4 is first expressed at E14.5 in epidermal spinous cells as they lose adhesion to the basement membrane, withdraw from the cell cycle, and commit to terminally differentiate. $\mathrm{Klf4}^{-/-}$embryos die perinatally as a consequence of the rapid water loss across the skin surface in the terrestrial environment. Conversely, ectopic expression of Klf4 in the epidermis from an inducible keratin 5 (K5) promoter accelerates barrier acquisition by approximately 1 day (12). Together, these animal models suggest that within a field of competence, Klf4 is both necessary and sufficient for epidermal terminal differentiation and subsequent barrier acquisition. Regulation of epidermal proliferation and differentiation is established in utero and maintained throughout life in a homeostatic balance. However, stresses, such as wounding or an inflammatory response, transiently tip this balance. We hypothesized that querying the factors necessary to establish this balance in the very sensitive in utero environment might also identify key regulators of this process when it is recapitulated in adult skin. We 
Table 1

Comparison of $\mathrm{KIf4}^{--}$and psoriatic skin expression profiles

\begin{tabular}{lccc} 
Gene symbol & Gene name & Psoriasis & KIf4-/- \\
ACPP & Acid phosphatase prostate & 3.0 & 3.9 \\
CTSHPC & Cathepsin C & 2.4 & 1.9 \\
CLCA2 & Chloride channel calcium activated 2 & 2.5 & 3.3 \\
DSC2 & Desmocollin 2 & 7.0 & 2.5 \\
EPRG & Epiregulin & 2.0 & 11.4 \\
ER01-like & ER01-like & 2.6 & 2.5 \\
EVA1 & Epithelial V-like antigen 1 & 4.0 & 1.8 \\
GJB2(CX26) & Gap junction protein, $\beta$ 2 & $>10$ & 3.4 \\
GJB6(CX30) & Gap junction protein, $\beta$ 6 & 5.2 & 3.4 \\
GK $\quad$ Glycerol kinase & 5.9 & 3.9 \\
HK2 & Hexokinase 2 & 2.6 & 3.4 \\
HIF1a & Hypoxia-inducible factor 1 $\alpha$ & 2.3 & 1.9 \\
IFITM1 & Interferon-induced transmembrane 1 & 2.2 & 1.8 \\
ILIR1N & Interleukin 1 receptor antagonist & 2.4 & 2.4 \\
MAD & MAX dimerization protein & 3.2 & 2.7 \\
SLC6A14 & Solute carrier family 6, member 14 & $>10$ & 3.3 \\
SPRR1B & Small proline-rich protein 1B & 4.6 & $>10$ \\
SPRR2A & Small proline-rich protein 2A & 5.9 & 5.0 \\
& & & \\
\hline
\end{tabular}

Levels of gene upregulation in psoriatic and $\mathrm{Klf4}^{-{ }^{--}}$skin. $\mathrm{Klf4}^{-/-}$expression levels were determined by microarray hybridization, and psoriatic expression profiling data are published results from Bowcock laboratory (2).

compared the transcriptional profile of the $\mathrm{Klf4}^{-{ }^{-}-}$newborn skin and psoriatic lesioned skin and identified connexin $26(\mathrm{Cx} 26)$ as one of the most highly upregulated genes $(10,13,14)$.

Cx26 is 1 of 8 connexin family members expressed in epidermis (15). Connexins homo- or heteromerize on the plasma membrane to form a connexon. Connexons on adjoining cells associate to form gap junctions and allow the passage of ions and small molecules between cells. Unpaired connexons or hemichannels allow the cell to release second messengers (16). Normally, Cx26 is expressed in proliferative epidermis during early embryonic development and wound reepithelization but downregulated to almost undetectable levels as terminal differentiation proceeds and Klf4 is expressed $(10,17-20)$. CX26 is one of the most highly upregulated genes in psoriatic plaques $(13,14)$. Hodgins and Salomon independently demonstrated with immunohistochemical studies that CX26 is not detected in normal and unlesioned skin but is expressed intensely at the cell periphery of keratinocytes in a psoriatic plaque $(21,22)$. Strong evidence for CX26 playing a role in epidermal differentiation also comes from human patients. Dominant-acting missense mutations in CX26 result in 5 distinct skin disorders (Vohwinkel syndrome, keratitis-ichthyosis-deafness syndrome, palmoplantar keratoderma with deafness, hystrix-like ichthyosis with deafness, keratopachydermia and constrictions of fingers and toes with deafness), which share the common feature of hyperkeratosis $(23,24)$. The dermatologic manifestation of human patients with CX26 mutations, together with the expression of CX26 in development, wound healing, and psoriasis, predict that the regulation of gap junctions is essential to normal epidermal differentiation.

This study tests our hypothesis that a common genetic pathway regulates barrier acquisition during embryonic development and after wound healing. Specifically, we examine the role of barrier reestablishment in the transition from the hyperproliferative state of keratinocytes during reepithelialization to the homeostatic bal- ance of proliferation and differentiation during remodeling of a wound. We demonstrate that Cx26 plays a linked causative role in barrier acquisition, wound healing, and promotion of an inflammatory state.

\section{Results}

Transcriptional profiling of Klf4-/- skin. To investigate the role of barrier disruption in psoriasis, we sought to identify common molecular pathways between $\mathrm{Klf4}^{-/-}$barrier-deficient skin and psoriatic plaques. $\mathrm{Klf4}^{-/-}$skin mRNA was profiled on microarray, and the compilation of genes upregulated 2-fold or greater was compared with a published expression profile of psoriatic skin from Bowcock's laboratory (Table 1) $(10,13)$. mRNA expression profiling cannot provide a comprehensive comparison of 2 tissues, since many changes occur at the protein level. Moreover, this transcriptional profile does not distinguish between direct targets of KLF4 and reactive changes due to barrier deficiency. However, changes in expression profiles provide an entry point to identify common regulatory pathways. As previously shown, genes encoding members of the small proline-rich (SPRR) protein family are significantly upregulated in both $\mathrm{Klf4}^{-{ }^{--}}$and psoriatic plaques (25-27). This upregulation of the SPRR genes has been previously observed as being part of a general stress response to a breach in barrier $(28,29)$. We observed in both samples upregulation of genes encoding proteins that activate the immune cells in preparation for bacterial invasion and oxidative stress secondary to barrier breach, including IL-1 receptor antagonist and hypoxia-inducible factor 1 , respectively. The common upregulation of the mitogen epiregulin may be the signal for the hyperproliferation observed in both psoriatic and barrier-deficient skin. In fact, ectopic expression of the closely related mitogen amphiregulin in the skin induces a psoriasiform-like response (30, 31 ). Thus, the comparison of the 2 tissue types presents a number of interesting candidates that either play a causative role in the barrier dysfunction or regulate the response to this state. We chose to focus our investigation on the gap junction protein $\mathrm{Cx} 26$ because of the potential biological significance of gap junctions in human disorders, wound healing, and development and its possible role in barrier acquisition $(15,23,24,32)$.

$K L F 4$ represses $C x 26$. During embryonic development, $\mathrm{C} \times 26$ is normally expressed in keratinocytes during the proliferative state and then downregulated at approximately E16.5 as differentiation proceeds and the barrier is acquired $(15,17)$. Barrier-deficient $\mathrm{Klf4}^{-/-}$ embryos do not downregulate $\mathrm{Cx} 26$, expressing 3-fold more than control littermates at E16.5, with continued high levels as E18.5 embryos and newborns. Conversely, transgenic mice, ectopically expressing Klf4, repressed the Cx26 expression earlier in development, with 1.6-fold lower expression than control littermates at E16.5. (Figure 1A). CX26 protein was expressed in suprabasal cells during normal embryonic development and retained this strong expression pattern in $\mathrm{Klf4}^{-/-}$newborn skin, with very weak expression in control littermates (Figure 1B). To examine whether Cx26 is a direct target of KLF4, we analyzed the regulation of Cx26 transcription. The minimal proximal promoter of $\mathrm{Cx} 26$ is active in keratinocytes, and cotransfection with Klf4 represses this activity in a dose-dependent fashion (Figure 1C). Computational analysis predicted $2 \mathrm{KLF}$ binding sites in the $\mathrm{Cx} 26$ proximal promoter sequence, and EMSAs showed that KLF4 binds directly to these sites (Figure 1D and Supplemental Table 1; supplemental mate- 
A

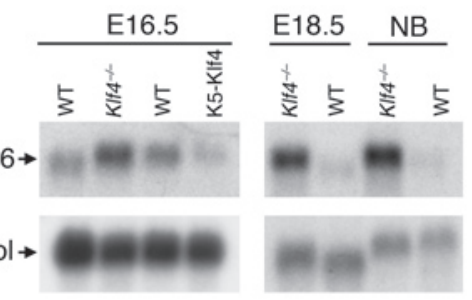

B
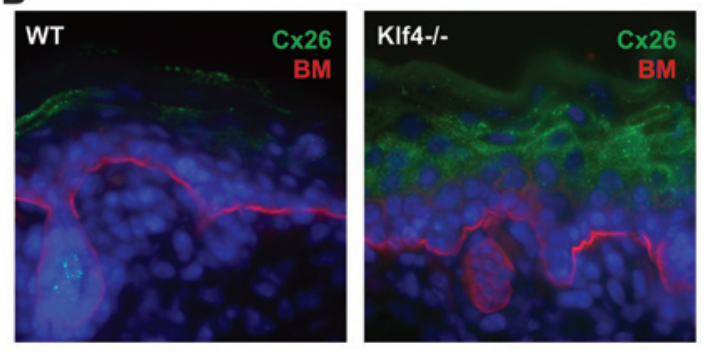

C

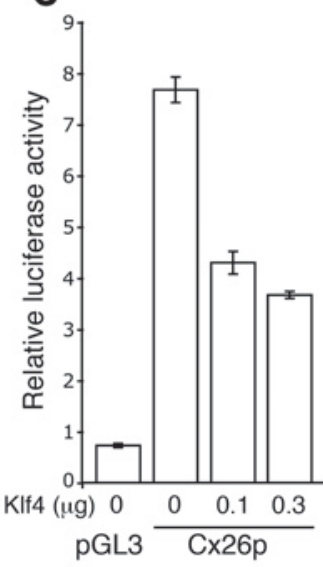

D

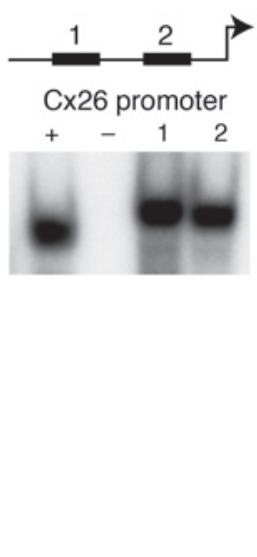

E

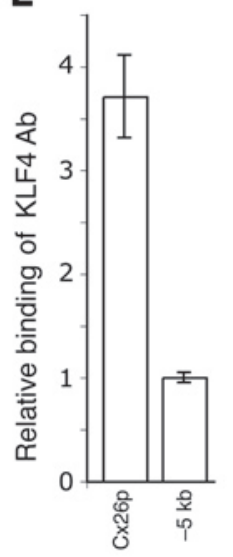

rial available online with this article; doi:10.1172/JCI27186DS1). To confirm that Cx26 promoter is an in vivo target of KLF4, we performed chromatin immunoprecipitation assays with a KLF4 antibody. Quantitative real-time PCR performed on specific KLF4 immunocomplexes demonstrated that the $\mathrm{Cx} 26$ proximal promoter region is enriched 3.7-fold relative to an amplicon $5 \mathrm{~kb}$ proximal to the Cx26 locus (Figure 1E). These results indicate that KLF4 binds directly to the $\mathrm{Cx} 26$ promoter to repress its transcription.

$C \times 26$ regulates epidermal barrier acquisition. To determine whether $\mathrm{Cx} 26$ has a primary role in the establishment of the epidermal permeability barrier, we phenocopied the expression of Cx26 observed in the $\mathrm{Klf4}^{-/-}$mice by ectopically expressing Cx26 from the human involucrin promoter (Inv) (Figure 2A) (33-35). Three separate lines of Inv-Cx 26 mice (F, K, and L) were generated that all demonstrated a consistent level of expression of Cx26. To generate an allelic series, we bred heterozygous mice to generate homozygous transgenic mice. We performed interphase FISH to determine that each line had a unique integration site. Subsequently, heterozygous and homozygous transgenic mice from a cross were distinguished by metaphase FISH as carrying 1 or 2 copies of the transgene, respectively (Figure 2B). In contrast to minimal expression in control newborn littermates, CX26 protein was expressed throughout the suprabasal cells in the heterozygous and homozygous Inv-Cx26 mice (Figure 2C). Confocal microscopy localized CX26 protein to the cell membranes (inset, Figure 2C). Homozygous Inv-Cx26

\section{Figure 1}

KLF4 regulates Cx26 transcription levels by binding directly to the proximal promoter. (A) Levels of Cx26 mRNA are relative to Klf4 expression. At E16.5, when the epidermal permeability barrier was first established, Klf4-/- and K5-Klf4 transgenic mice express 3.0-fold higher and 1.6-fold lower levels of Cx26 mRNA than wild-type littermates, respectively. The upregulation of Cx26 persists in $\mathrm{Klf4}^{-/-}$mice at E18.5 and as newborns (NB). (B) CX26 protein is weakly expressed in the suprabasal layers of newborn wild-type skin and strongly expressed in $\mathrm{KIf4}^{-/}$epidermis. Basement membrane (BM) is marked with $\alpha 6$ integrin staining. Magnification, $\times 40$. (C) KLF4 protein represses minimal Cx26 promoter in a dose-dependent manner. Cx26 promoter (Cx26p) yields activation 8 -fold greater than promoterless pGL3 vector. This activity is repressed in a dose-dependent fashion by cotransfection with Klf4. (D) KLF4 binds sequences in the proximal Cx26 promoter. Computational analysis predicted 2 KLF4 binding sites in the minimal Cx26 promoter. As shown by EMSA, both sites are bound by KLF4. +, positive control for KLF4 binding site; -, mutated KLF4 site. (E) KLF4 binds directly to the proximal Cx26 promoter. Quantitative realtime PCR performed on specific KLF4 chromatin immunocomplexes demonstrated that the Cx26 promoter region is enriched 3.7-fold relative to an amplicon $5 \mathrm{~kb}$ proximal to the Cx26 locus. Results for all samples are normalized to input DNA.

transgenics expressed twice as much CX26 mRNA and protein as heterozygous mice (Figure 2, D and E). Although connexin gene expression can be coordinately regulated under physiologic conditions, heterozygous and homozygous Inv-Cx26 transgenics express normal levels of the other epidermal connexins (Cx30, -30.3, -31.1, and -43) (data not shown) (15).

Newborn homozygous Inv-Cx26 transgenic mice display taut, dry, shiny skin and die perinatally ( $<12$ hours). Heterozygous InvCx26 mice display mild erythema and vascular prominence with a normal survival rate (Figure 3A). At E18.5 when littermates have fully established the barrier, homozygous Inv-Cx26 mice exhibit profound barrier impairment, as demonstrated by percutaneous dye penetration, while heterozygotes have a mild defect, visualized by the light blue coloration (Figure 3B) (11). Homozygous Inv-Cx26 mice display a high rate of transepidermal water loss, with the heterozygous mice demonstrating a moderate increase as compared with control mice (Figure 3C). Translating this water loss across the skin surface, homozygous Inv-Cx26 mice lose more than $7 \%$ of their body weight within 6 hours. Heterozygous mice have only a slight decrease in weight, consistent with their postnatal survival (Figure 3D). In summary, persistent expression of Cx26 during development, as observed in barrier-deficient $\mathrm{Klf4}^{-{ }^{--}}$mice, is sufficient to impair barrier acquisition in utero.

Ectopic Cx26 expression increases junctional communication and stimulates hyperproliferation. Homozygous Inv-Cx26 newborn skin is mildly acanthotic and hyperkeratotic, with a thicker, more compact cornified layer (Figure 4, A and B). Epidermal differentiation markers K14, K10, Inv, and loricrin expression profiles were similar among all transgenics and littermates (Supplemental Figure 1A). K6, a marker of hyperproliferative epidermis, including psoriatic plaques, was expressed suprabasally in homozygous Inv-Cx26 mice (Figure 4, C and D, and Supplemental Figure 1B). Consistent with this, homozygous newborns displayed the highest BrdU incorporation after a pulse-label (20\%) as compared with wild type (11\%), with the heterozygous displaying an intermediate rate (15\%) (Figure 4, E and F, and Supplemental Figure 1C). In all cases, proliferation was restricted to the basal layer. Electron microscopy of 
A

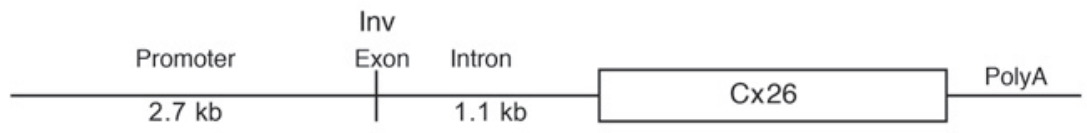

B
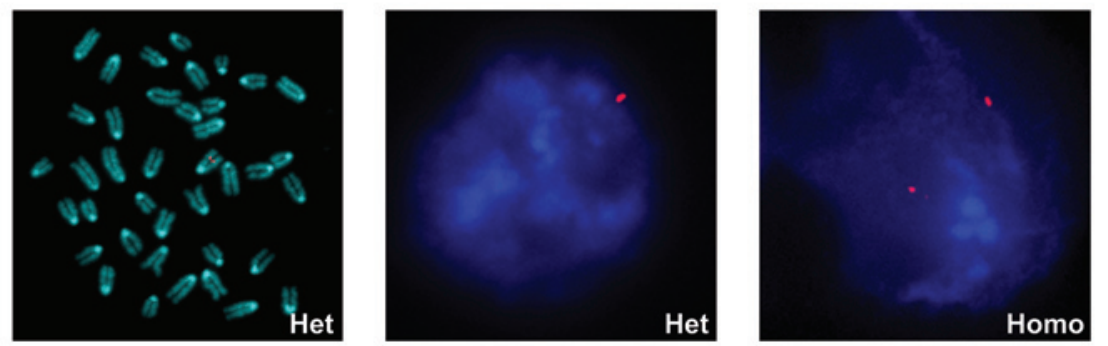

C
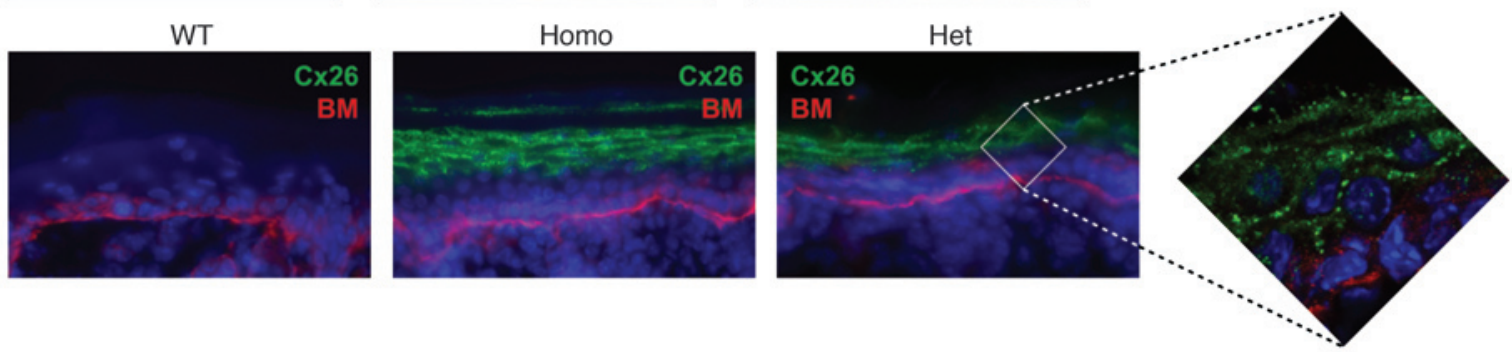

D
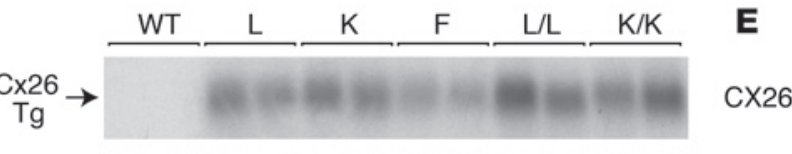

X26 $\rightarrow$
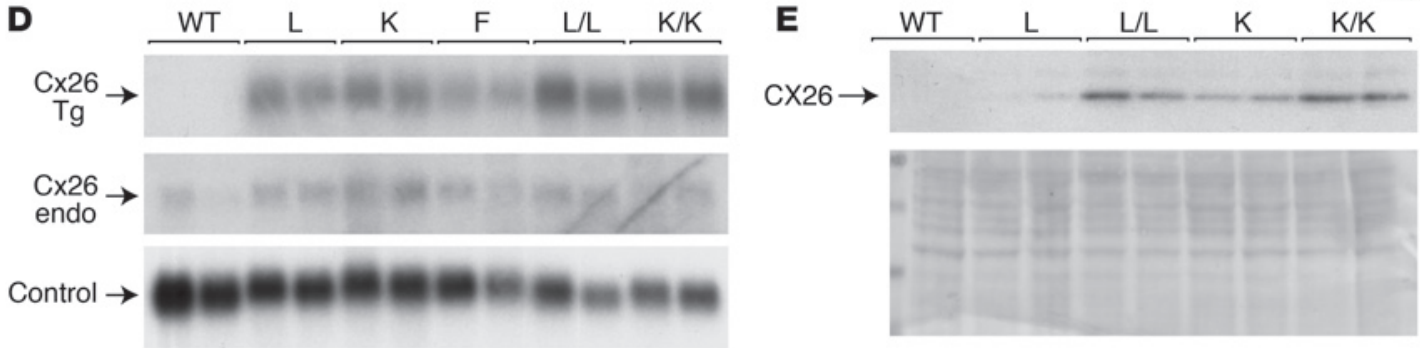

Figure 2

Molecular characterization of the Inv-Cx26 transgenic mice. (A) Schematic representation of the Inv-Cx26 transgene. The complete murine Cx26 cDNA sequence was cloned downstream of the Inv regulatory sequences. (B) Genotyping of transgenic mice by interphase FISH with distinct chromosomal insertion sites (representative data for 1 line). Metaphase FISH was used for all further studies to genotype littermates resulting from crossing 2 heterozygous (Het) mice: heterozygous transgenic mice (1 hybridization signal) and homozygous (Homo) transgenic mice (2 hybridization signals). (C) CX26 immunohistochemistry demonstrated expression in the suprabasal layer of newborn heterozygous and homozygous Inv-Cx26 transgenic mouse skin at the membrane (inset: confocal image). (D) Expression levels of transgenic and endogenous (endo) Cx26 mRNA in 3 different lines of Inv-Cx26 mice, both heterozygotes (L, K, and F) and homozygotes (L/L and K/K). (E) Expression of CX26 protein in heterozygous and homozygous Inv-Cx26 mice. Homozygote L/L and K/K Inv-Cx26 mice expressed twice as much protein as their respective heterozygote $L$ and $K$ littermates. Magnification, $\times 100$ (B, inset $C), \times 40$ (C).

homozygous Inv-Cx26 newborn skin revealed an increased number of gap junctions in the suprabasal layers of the epidermis, with no other abnormalities (Figure 4, G and $\mathrm{H}$ ). In vivo intercellular gap junction communication was assessed specifically by a neurobiotin dye injection into an incision on the dorsal skin. Dye transfer was greatly augmented in homozygous Inv-Cx26 skin, demonstrating the physiologically significant increase in junctional communication (Figure 4, I and J). Tight junctions appeared to be functionally intact in the Inv-Cx26 skin (data not shown) (36). Together, these results demonstrate that increased epidermal CX26 expression produces an increase in functional gap junctions, stimulates hyperproliferation, and impairs barrier acquisition.

Ectopic Cx26 expression impairs epidermal remodeling of wounds. In the first week of life, heterozygous Inv-Cx26 mice develop mild scaling and desquamation of the skin, particularly in the axillary areas, which are susceptible to frictional trauma. These lesions progress and become hyperkeratotic plaques. Lesions that circumferentially involve a limb and occlude the lymphatic return often cause the affected limb to become massively swollen. Circumferential hyperkeratotic lesions involving the tail lead to distal necrosis. Both of these phenotypes are reminiscent of the hyperkeratotic plaques and autoamputation of digits seen in CX26-related Vohwinkel syndrome. As adults, heterozygous Inv-Cx26 mice develop similar hyperkeratotic lesions on the dorsal surface behind the ears and on the entire ventral surface, traumatized by scratching and rubbing against the cage floor, respectively (Figure 5A). Histologically, the nonlesioned skin demonstrated mild acanthosis and hyperkeratosis, typical of mild barrier impairment. Immune infiltration in these nonlesioned areas of the skin was not observed. The reepithelialized lesioned areas of the epidermis displayed parakeratosis, suprapapillary formation extending deep into the dermis with thinner plates overlying dilated vessels, hyperkeratosis, hypogranulosis, and acanthosis. Neutrophils were present in both the suprabasal and cornified layers of the epidermis (Figure 5B). The 
A
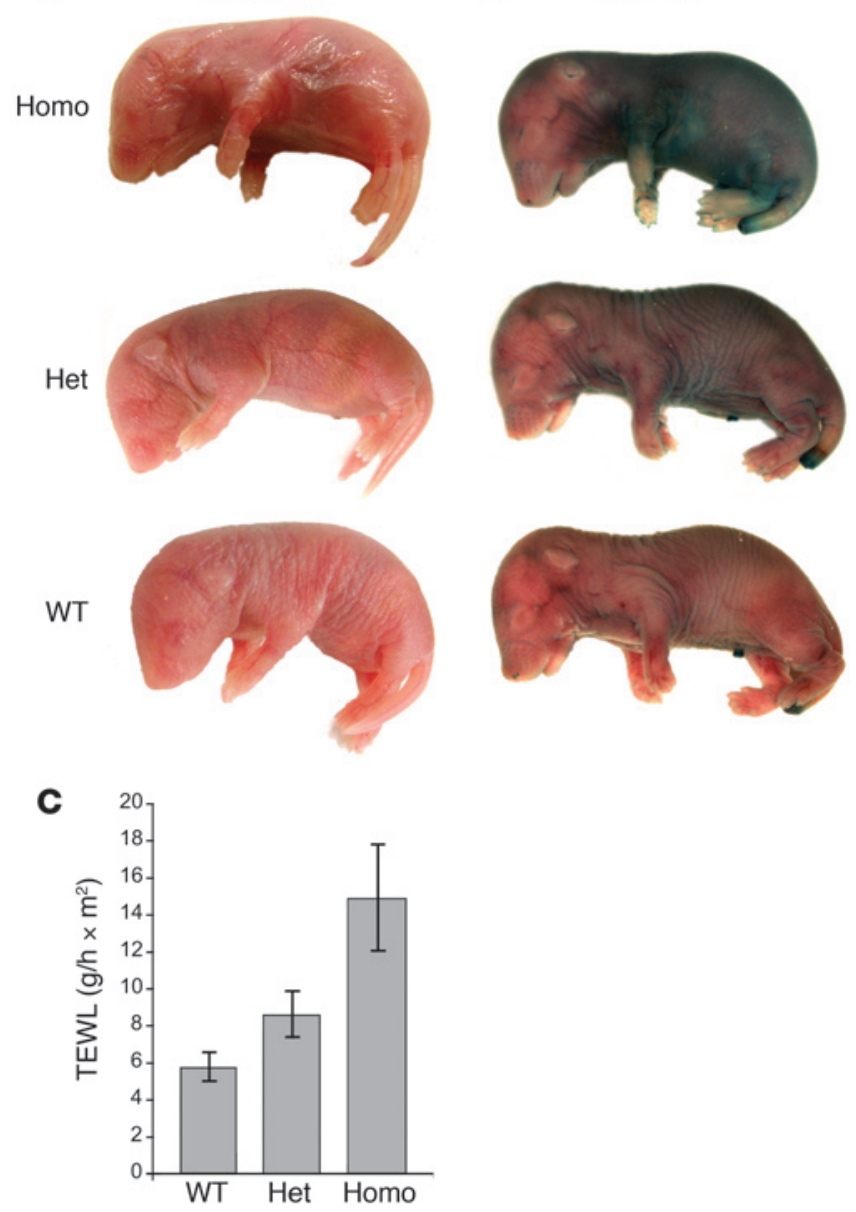

D

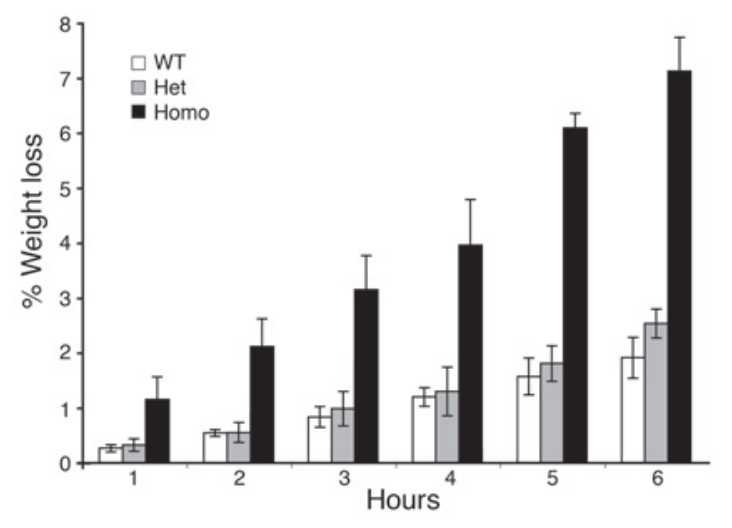

lesioned areas displayed a lymphocytic cell infiltrate, staining positively for both CD4 and CD8 (Figure 5C).

To evaluate whether wounding is the initiating event in generating the hyperkeratotic lesions, we created $5-\mathrm{mm}$ punch biopsy wounds on the unaffected dorsal skin, where the mice cannot scratch. During the first week, wild-type and heterozygous InvCx26 mice displayed similar progression of the wounds. On a macroscopic level, the wounds of all control mice had healed by 2 weeks compared with $21 \%$ and $42 \%$ of the heterozygous InvCx26 mice at 2 and 3 weeks, respectively. Heterozygous Inv-Cx26 mice displayed persistence of a scaly plaque up to 6 weeks after the biopsy (Figure 6A). Histologically, at day 7, a migrating epithe-

\section{Figure 3}

Inv-Cx26 transgenics exhibit a barrier deficiency. (A) Newborn homozygous Inv-Cx26 transgenic mice display taut, dry, shiny skin. Heterozygous Inv-Cx26 mice displayed mild erythema and vascular prominence. (B) E18.5 Inv-Cx26 homozygous mice have an impaired epidermal barrier. Heterozygous Inv-Cx26 mice exhibited a mild barrier defect, distinguishable from that of wild-type littermates. Establishment of epidermal barrier was studied with a whole-mount dye penetration assay. Blue represents areas without a functional barrier, in which the dye has penetrated percutaneously. White areas have acquired barrier and are impermeable to the dye penetration. (C) Homozygous InvCx26 skin exhibited significantly increased transepidermal water loss (TEWL). Heterozygous Inv-Cx26 skin exhibited mild transepidermal water loss. (D) Homozygous Inv-Cx26 mice exhibited severe weight loss over 6 hours. Weight loss of heterozygous Inv-Cx26 mice was not statistically different from that of wild-type controls.

lial edge was evident in both control and heterozygous Inv-Cx26 mice. In control mice, a normal stratified epidermis was reestablished by day 14. In contrast, epidermis of heterozygous Inv-Cx 26 mice failed to acquire a normal structure and displayed acanthosis, hyperkeratosis, hypogranulosis, and parakeratosis (Figure 6, $\mathrm{B}$ and $\mathrm{C}$ ). These histological abnormalities of heterozygous InvCx26 wounds were evident even if macroscopically the ichthyotic scab resolved after 2 or 3 weeks. Similar lesions were also seen after barrier disruption by tape strip injury to the epidermis (data not shown). Immunofluorescence studies in control mice demonstrated CX26 expression in the epidermal proliferative zone near the wound margin at 7 days after wounding, returning to undetectable levels by day 21. In heterozygous Inv-Cx 26 mice, CX26 expression was present at the wound margins and in the new epithelium at both 7 and 21 days after wounding (Figure 6D). Keratinocytes in the reepithelialized skin of the heterozygous Inv-Cx26 mice remained in a hyperproliferative state as compared with controls, as determined by an increased number of BrdU-positive cells in the epidermal basal layer (Figure 6E). The reepithelialized areas of heterozygous Inv-Cx26 mice demonstrated a significant infiltration of inflammatory cells, including neutrophils and lymphocytes, identical to the pattern observed in the self-induced wounds shown in Figure 5 (data not shown). This immune infiltration was not observed in nonlesioned skin or control wounds at 21 days. After reepithelialization, persistent expression of CX26 kept the keratinocytes in a hyperproliferative state instead of transitioning to the proper balance of proliferation and differentiation.

Cx26 regulates ATP release. To elucidate the mechanism by which CX26 expression regulates epidermal differentiation and contributes to the pathogenesis of the hyperkeratotic lesions, we studied the release of ATP from keratinocytes. Previous studies have implicated ATP release through CX26 hemichannels in intestinal epithelial disease (37). The ATP release by heterozygous Inv-Cx26 keratinocytes was significantly higher than that by controls (92 nM versus $48 \mathrm{nM}$ ATP; $P<0.002)$. In both control and heterozygous Inv-Cx26 keratinocytes, ATP release was suppressed with carbenoxolone, a gap junction and hemichannel blocker $(28$ nM ATP for both genotypes) (Figure 7A). To further understand how this might affect barrier acquisition, we examined the calcium response of wild-type keratinocytes to ATP. Single-cell measurements of keratinocytes loaded with the calcium ratiometric dye fura-2 demonstrated a strong increase in intracellular calcium in response to ATP stimulation. The ATP-induced calcium flux in keratinocyte was blocked by pyridoxalphosphate-6-azophenyl- 


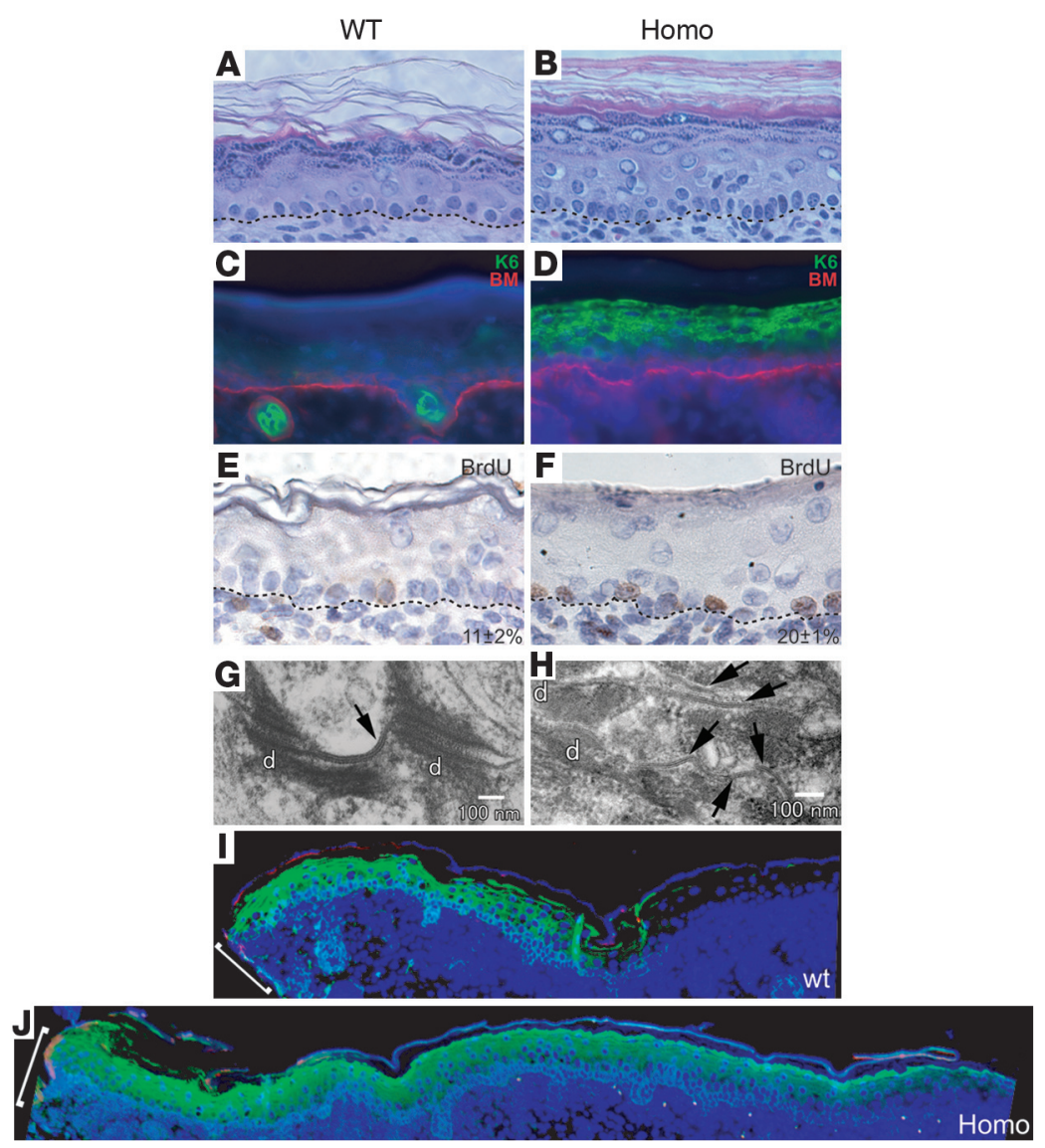

\section{Figure 4}

Histology of homozygous Inv-Cx26 mice with hyperkeratosis, hyperproliferation, increased number of gap junctions, and enhanced in vivo dye transfer. ( $\mathbf{A}$ and B) Histology of homozygous Inv-Cx26 skin reveals increased number of spinous cell layers with more compact stratum corneum. The dotted lines mark basement membrane. (C-F) Hyperproliferation in homozygous Inv-Cx26 mice with upregulation of K6 suprabasally and increased number of BrdU-positive cells. The dotted or $\alpha 6$-stained red lines mark basement membrane. ( $\mathbf{G}$ and $\mathbf{H}$ ) Increased gap junctions in homozygous Inv-Cx26 skin. Arrows indicate gap junctions. d, desmosomes. (I and $\mathbf{J}$ ) In vivo dye transfer experiments with neurobiotin (green) and rhodamine dextran (red) demonstrated increased junctional communication in homozygous Inv-Cx26 skin. The white bracket marks the wound incision. Only 1 side of wound is shown. Magnification, $\times 40(\mathbf{A}-\mathbf{F}), \times 10$ (I and J). Scale bars: $100 \mathrm{~nm}$ ( $\mathbf{G}$ and $\mathbf{H})$.
2 ', $4^{\prime}$-disulfonic acid (PPADS), an antagonist of purinergic receptors (Figure 7B). Thus, CX26 hemichannels release ATP to activate purinergic receptors and regulate keratinocyte calcium flux.

\section{Discussion}

The studies presented here demonstrate that CX26 must be downregulated during in utero development to acquire the skin's permeability barrier and after wounding to progress epidermal remodeling. Spatial and temporal expression patterns show that KLF4 is expressed concomitant with the downregulation of Cx26 $(10,17-20)$. We show that KLF4 binds directly to the proximal Cx26 promoter, repressing its transcription. Recent reports have focused on similar effects of KLF4 as a repressor. KLF4 acts as a context-dependent oncogene by binding directly to the p53 promoter and repressing its expression (38). KLF4 also acts as a myogenic repressor to oppose serum response factor activation of smooth muscle gene expression both during development and in response to vascular injury (39). Generating additional antibodies for chromatin immunoprecipitation or targeting a tagged version of Klf4 into the endogenous locus will aid further analysis to identify KLF4's direct targets.

The proper balance of epidermal proliferation and differentiation must be established during development and then recapitulated during reepithelialization of a wound. Our studies suggest that there is a molecular connection between these 2 decisions and that junctional communication through connexons similarly regulates both processes. Persistent CX26 expression arrests both epidermal development and wound healing in a hyperproliferative state. Our model posits that reestablishment of the epidermal barrier marks the transition from reepithelialization to remodeling of a wound and a return to the homeostatic balance of proliferation and differentiation. The barrier impairment of heterozygous InvCx26 mice tips this balance, retaining the keratinocytes in a hyperproliferative state after reepithelialization and inhibiting progress to the proper remodeling of the wound.

Previous investigators have remarked upon the similarities between acute reepithelialization during wound healing and psoriasis because of the observed hyperproliferation of keratinocytes and infiltration of immune cells (40). Unlesioned areas of Inv-Cx26 heterozygotes are mildly acanthotic but otherwise unremarkable, with no signs of immune cell infiltration. Reepithelialized InvCx26 heterozygous skin displays parakeratosis, suprapapillary formation extending deep into the dermis with thinner plates overlying dilated vessels, hyperkeratosis, hypogranulosis, and acanthosis. Neutrophils are present in both the living and cornified layers of the epidermis. As well, lymphocytes are resident in the epidermis. These Inv-Cx26 heterozygous transgenics show a similarity to the isomorphic phenomenon observed in patients in that wounding elicits many of the hallmark features of psoriasiform dermatitis.

Barrier disruption stimulates keratinocyte proliferation and cytokine induction $(8,9)$. The immune cell activation associated with a disrupted barrier may be a result of mitogenic signals acting on the immune cells and/or increased antigen presentation with skin penetration. Here we elucidate a possible intermediate step in the process by which a breached barrier signals to activate immune cells. Expression profiling and immunohistochemical data have 

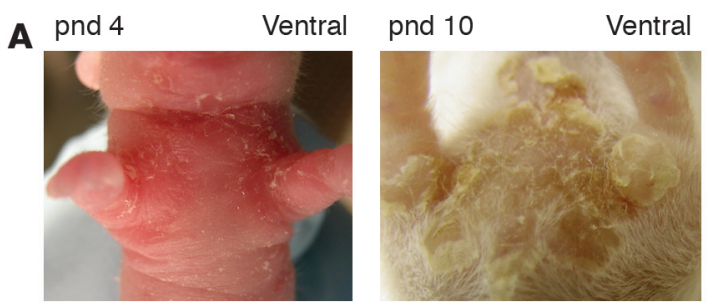

$$
\text { Adult Dorsal }
$$

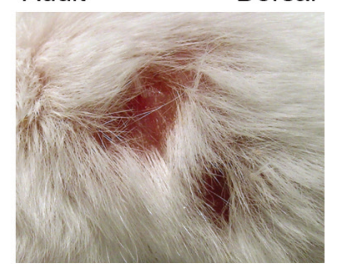

B

WT

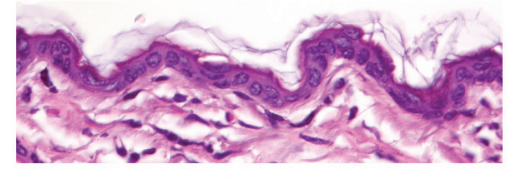

Het lesioned
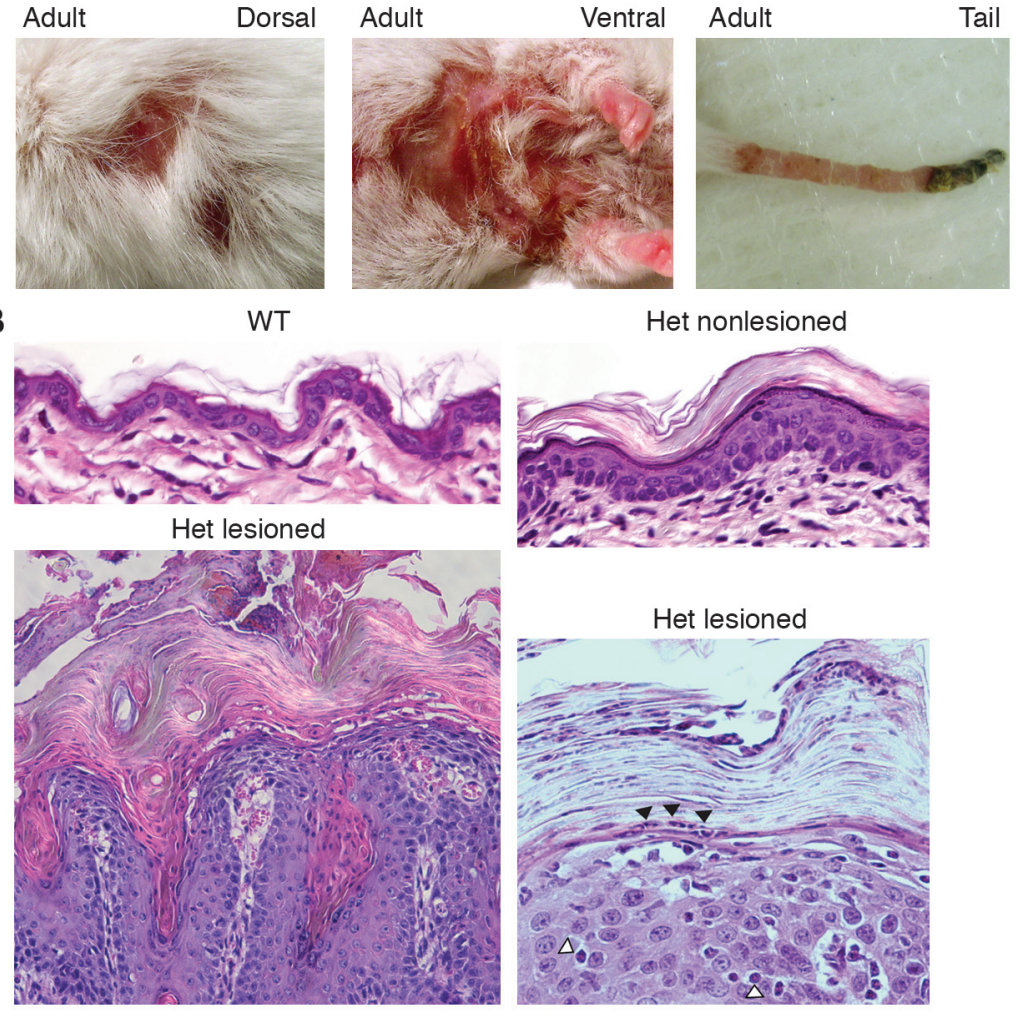

Het nonlesioned
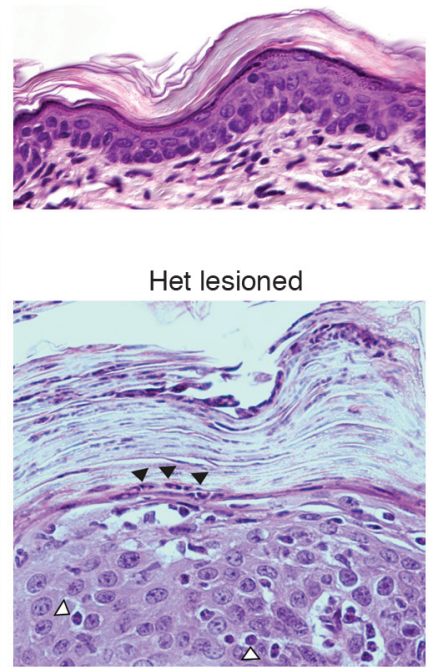

C

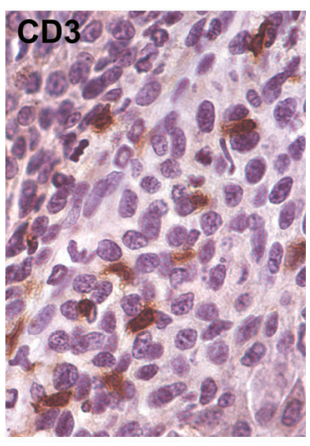

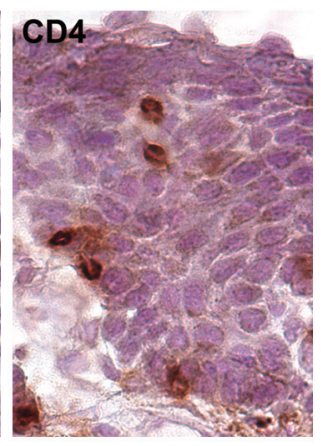

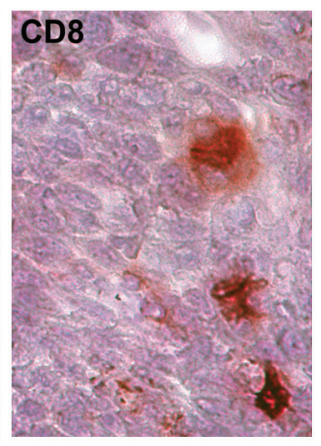

Paw

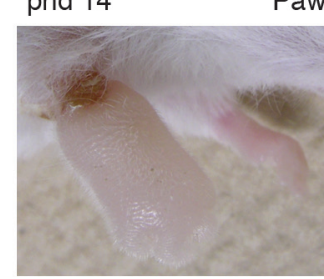

Tail

(a)
wheads) and horny layers (filled arrowheads). (C) Lymphochytic infiltration in heterozygous Inv-Cx26 lesioned skin of $\mathrm{CD}^{+}, \mathrm{CD}^{+}$, and $\mathrm{CD} 8^{+}$cells. All sections were counterstained with hematoxylin. Magnification, $\times 20$ (B, lower left panel), $\times 40$ (B and C).

dermis $(43,44)$. Furthermore, keratinocyte release of ATP exacerbates inflammation by activating $\mathrm{CD} 9^{+}$Langerhans cells, as observed in response to irritant chemicals $(45,46)$. Hence, connexonmediated ATP release by keratinocytes can affect intracellular calcium concentrations, regulate epidermal proliferation and differentiation, and exacerbate the skin inflammatory response modulated by Langerhans cells.

The release of ATP via connexons may also be a contributing factor in the pathogenesis of connexin-related skin disorders. Dominantly inherited missense mutations in $\mathrm{Cx} 26$ and $\mathrm{Cx} 30$ result in multiple overlapping skin disorders characterized by hyperkeratosis $(47,48)$. CX26 and CX30 share $89 \%$ amino acid similarity and are directly adjacent on human chromosome 13. Both Cx26 and Cx30 are upregulated in $\mathrm{Klf4}^{--}$and psoriatic skin (Table 1). Functional studies demonstrated that 2 different missense mutations in CX30 that underlie human skin disorders result in proteins with increased hemichannel release of ATP (49). It is intriguing to speculate that the dominant-acting CX26 mutations may similarly result in increased hemichannel release of ATP due to increased protein levels or activity. Ectopic keratinocyte expression of normal CX26 protein has phenocopied some of the skin phenotypes of these human CX26 disorders. However, we do not observe the strong palmoplantar involvement observed in the human disorders, which might result either from transgene expression level or inherent differences between mice and humans.

previously demonstrated that CX26 is highly upregulated in psoriatic plaques. ATP release from CX26 hemichannels can activate purinergic receptors, found both on keratinocytes and Langerhans cells $(41,42)$. In keratinocytes, the increased release of ATP from CX26 hemichannels initially results in a rise of intracellular calcium. However, continual stimulation of keratinocytes by ATP depletes the intracellular calcium stores and desensitizes the ATP receptors from responding to the proper differentiation cues (43). Thus, while elevation of intracellular calcium levels normally promotes the differentiation process, excessive ATP stimulation inhibits terminal differentiation and barrier recovery in the epi-
Animal models for psoriasis, including those used in the recent studies of epidermis-specific loss of c-Jun and JunB and ectopic keratinocyte expression of activated Stat 3 or TGF- $\beta 1$, expand the links between the keratinocyte and immunologic causes of this complex disorder $(40,50,51)$. It will be interesting to test whether these animal models have impaired barrier recovery or altered gap junction communication to coalesce the keratinocyte contribution of psoriasis.

Identification of genes upregulated in barrier-deficient epidermis playing a significant role in inflammatory disorders extends beyond Cx26. Although the histologic presentation and type of 
A

(Day)

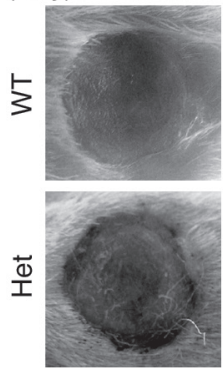

3

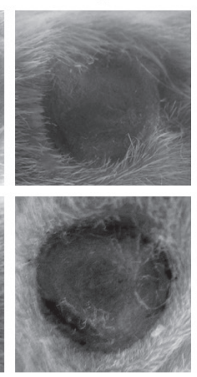

6

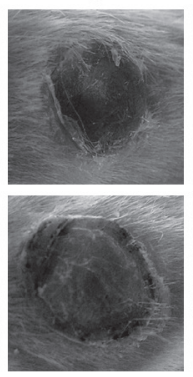

10

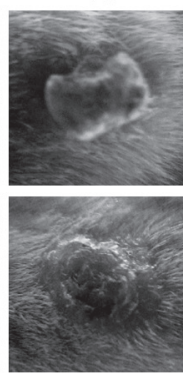

15

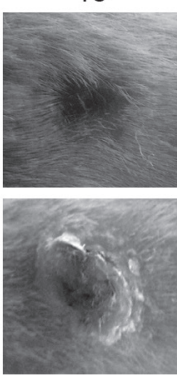

21

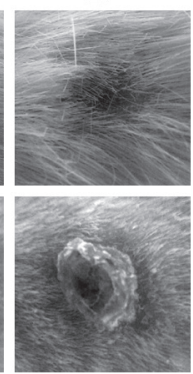

29

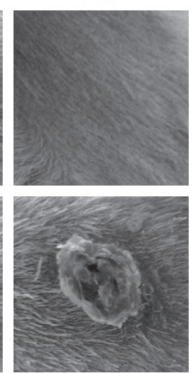

36

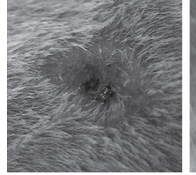

43
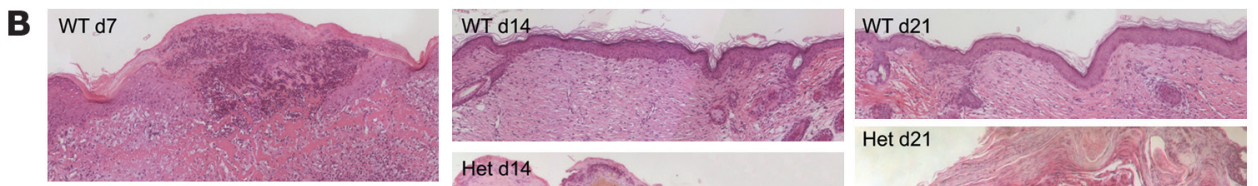

Het d21
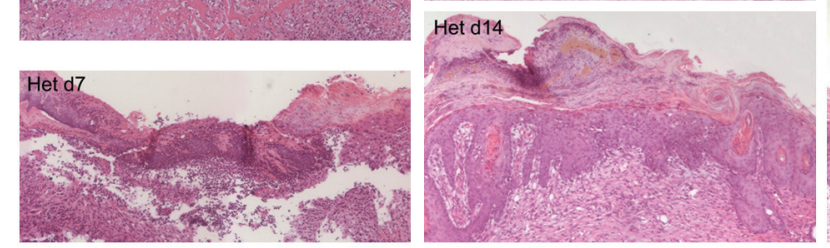

C
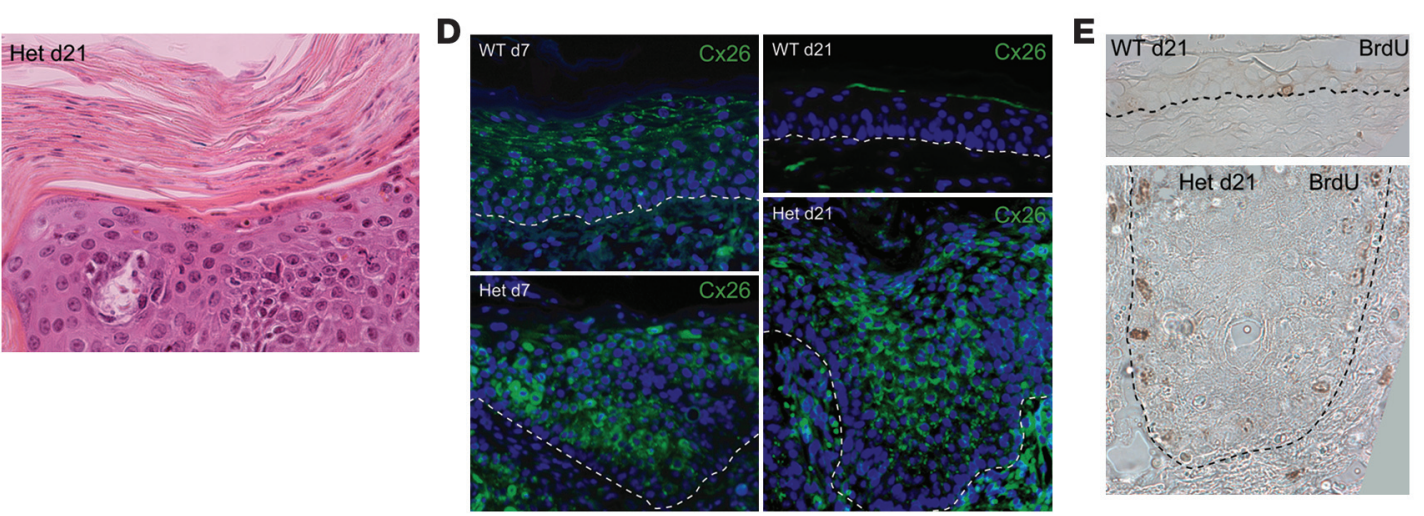

\section{Figure 6}

Heterozygous Inv-Cx26 mice reepithelialize wounds but fail to remodel and heal the wounds. (A) Gross appearance of 5-mm punch biopsy wound healing in wild-type control and heterozygous Inv-Cx26 mice over time. (B) Histology at 7, 14, and 21 days after wounding of heterozygous Inv-Cx26 mice. (C) Higher magnification of heterozygous Inv-Cx26 skin demonstrates hypogranulosis and parakeratosis. (D) Expression of CX26 protein 7 and 21 days after wounding on wild-type and heterozygous Inv-Cx26 mice. In control wounded skin, Cx26 is expressed in wound edge migrating epidermis but was undetectable after wound closure and remodeling. The heterozygous Inv-Cx26 epidermis retained CX26 expression even after reepithelialization. Basement membrane is marked by the dashed white line. (E) Heterozygous Inv-Cx26 epidermis remains hyperproliferative, as demonstrated by BrdU staining, even after reepithelialization. BrdU-positive cells were found in the basal layer. Basement membrane is marked by the dashed black line. Magnification, $\times 10$ (B), $\times 40(\mathbf{C}-\mathbf{E})$.

immune cell activation are very different for psoriasis and atopic dermatitis, both exhibit barrier deficiency proportional to pathologic severity. In our screen, we also identified the cytokine thymic stromal lymphopoietin (TSLP) as highly upregulated (>10-fold) in barrier-deficient $\mathrm{Klf4}^{-/-}$skin. TSLP is highly upregulated in keratinocytes of patients with atopic dermatitis and activates dendritic cells (52). Recent experiments have shown that transgenic expression of TSLP is sufficient to initiate atopic dermatitis and allergic airway inflammation or asthma when expressed in the skin and lung, respectively (53-55). The high-level expression of TSLP in barrier-deficient skin suggests that the breach is "sensed" and TSLP is expressed to mature the required immune cells. Interestingly, patients with Netherton syndrome, a congenital epidermal bar- rier defect, also develop atopic dermatitis (56). Moreover, a coding polymorphism in the gene underlying Netherton syndrome shows significant association with atopic dermatitis (57). Although the underlying causative polymorphism has not been identified precisely, both atopic dermatitis and psoriasis both show strong linkage to the epidermal differentiation complex, a cluster of genes encoding proteins that regulate and build the epidermal barrier $(6,58)$. Interestingly, of the genes upregulated in both $\mathrm{Klf4}^{---}$and psoriatic skin, only the Sprr genes are also upregulated in barrierdeficient newborn homozygous Inv-Cx26 skin. This suggests that KLF4 may regulate additional pathways in common with epidermal changes in inflammatory disorders that are independent of regulating CX26 levels. Based on these findings and our current 

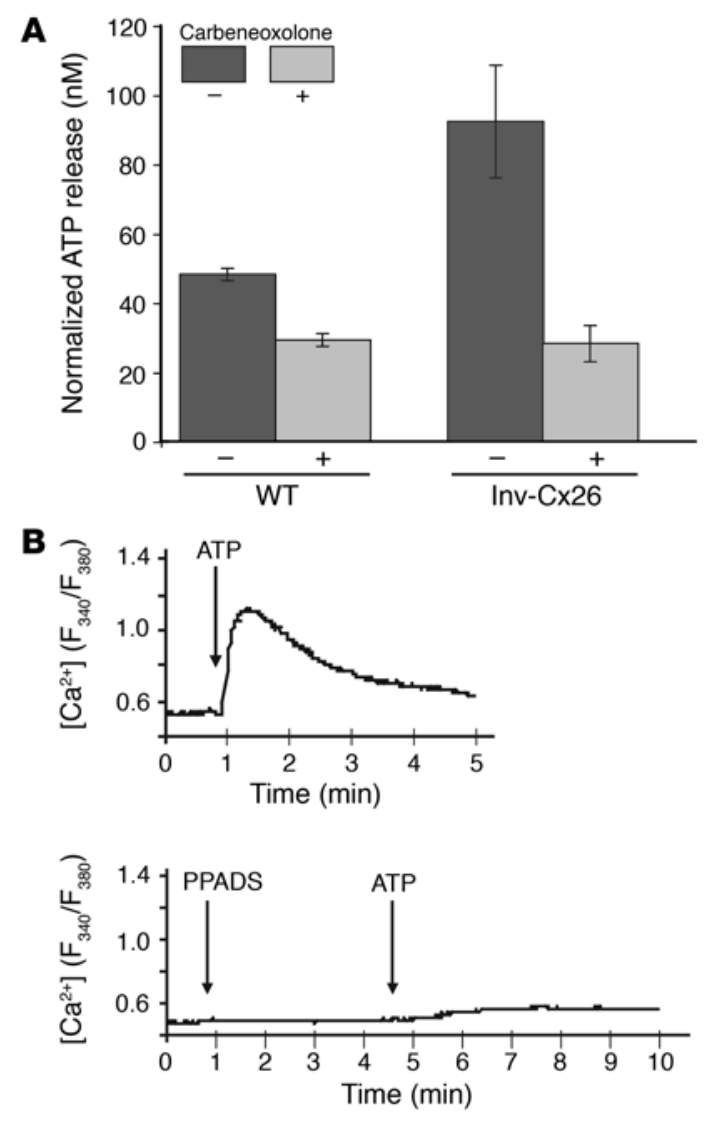

results, we posit that impaired barrier or barrier recovery may contribute to the progression of inflammatory skin disorders.

Our results suggest that therapies targeted to enhancing barrier acquisition may prove beneficial for resolving epithelialized hyperkeratotic lesions, such as psoriatic plaques. For example, studies in rodents have demonstrated that corticosteroids accelerate epidermal barrier acquisition, which may contribute to their beneficial effect on treating inflammatory disorders, independent of their immunosuppressive effects $(11,59)$. Previous studies on the role of connexins have demonstrated that downregulation of $\mathrm{Cx} 43$, either through genetic deletion or antisense treatment, resulted in accelerated wound closure $(60,61)$. Similarly, we propose that decreasing Cx26 levels of epithelialized lesions may provide a therapeutic benefit by reestablishing the epidermal barrier and modulating the skin inflammatory response. Our model suggests that there is a positive feedback loop between barrier recovery and inflammatory response and that the best treatments will act to both enhance epidermal barrier recovery and suppress immune response.

\section{Methods}

Generation of Inv-Cx26 construct and mice. Complete murine Cx 26 cDNA was amplified by PCR from American Type Culture Collection clone MGC18706 with primers that contained NotI sites at their $5^{\prime}$ ends. This Cx26 amplicon was digested with NotI and inserted into the unique NotI site in the human Inv promoter $(3.9 \mathrm{~kb}$; promoter provided by Joseph Carroll and Lorne Taichman, State University of New York at Stony Brook, Stony Brook, New York, USA) vector (pH3700-pL2). Orientation of the cDNA was determined by PCR and confirmed with sequencing. The INV-Cx26 construct was purified by $\mathrm{CsCl}$ gradient (Lofstrand). Thirty micrograms

\section{Figure 7}

Connexons regulate extracellular ATP release to control intracellular calcium levels. (A) Keratinocytes release ATP into media dependent on connexon function. Heterozygous Inv-Cx26 keratinocytes release twice as much ATP as control littermates. Release of ATP from both heterozygous Inv-Cx26 and wild-type keratinocytes is repressed by connexin blockers. (B) Addition of ATP fluxes calcium into keratinocytes. Pretreatment of keratinocytes with purinergic receptor antagonist PPADS blocks calcium response to ATP.

were digested with SalI (New England Biolabs) to free the insert, purified (with a QIAGEN kit), and injected into FVB/N 1-cell eggs following standard pronuclear injection. Positive founders were identified by PCR and maintained on $\mathrm{FVB} / \mathrm{N}$ background. DNA was isolated by standard techniques. All studies on animals were approved by the National Human Genome Research Institute Animal Care and Use Committee and performed following their recommendations. Housing and breeding followed the guidelines in use in our Association for Assessment of Laboratory Animal Care-accredited animal facility.

RNA isolation and Northern blot analyses. K5-Klf4 and Klf4-/- embryos and newborns were identified by genotyping as previously described $(10,12)$. Dorsal back skin was isolated by dissection, snap-frozen, pulverized, and homogenized in Trizol (Invitrogen Corp.), and RNA was extracted following the manufacturer's recommendations. For microarray studies, samples were purified with an RNeasy kit (QIAGEN). cDNA, labeled with Cy3 or Cy5 dUTP (Amersham Biosciences), was made from $30 \mu \mathrm{g}$ total RNA. cDNA microarray slides were purchased from Affymetrix (version MU430 2.0). Slides were analyzed on an Agilent scanner and evaluated with IPLab software (version 3.2.4; Scanalytics). Blots were hybridized with $C x 26$ antisense probe (bp 804-1413 of BC013634) or with GAPDH probe or K1 as loading controls for Klf4 and Cx26 transgenics, respectively. Transgenic and endogenous Cx26 transcripts were 1.0 and $3.0 \mathrm{~kb}$, respectively. Signals were quantified using Molecular Dynamics Inc. PhosphorImager and IQ analysis software (version 1.2; Amersham Biosciences).

Cell transfections, EMSAs, and chromatin immunoprecipitation assays. The region from -267 to +20 relative to the transcription start site of mouse Gjb2/Cx26 (defined by BY307234) (Chr14:49092354-49092640) was amplified by PCR and cloned into a promoterless pGL3 Basic vector expressing firefly luciferase (Promega). The complete coding sequence of KLF4 (bp 273-1800, GenBank accession number U20344) was amplified by PCR and cloned into pcDNA3 (Promega). SP-1 cells, initiated mouse keratinocytes, were cultured and transfected under previously described conditions, including Renilla luciferase plasmid (pRL-SV40) as control for transfection efficiency (Promega) (26). The DNA binding domain of KLF4 was cloned into TOPO His6 pET100 vector (amino acid 308-474 of S405921) and purified according to the supplier's recommendations for induction with $2 \%$ ethanol and $0.5 \mathrm{mM}$ isopropyl- $\beta$-D-thiogalactopyranoside (IPTG) at $30^{\circ} \mathrm{C}$ for 1 hour and purification under native conditions (Invitrogen Corp.). The Cx26 proximal promoter sequence with KLF binding sites shown in bold is supplied as Supplemental Table 1. Chromatin immunoprecipitation with KLF4 antibody (H-180; Santa Cruz Biotechnology Inc.) was performed as published previously (62). The primer sequences used for quantitative PCR of immunocomplexes were Cx26F: 5'-CGAGTAGCTGGGACTTGGAG-3' and Cx26R: 5'-CGAGCTCTCCTGGAGCCTA-3' and -5kbF: 5'-TCAGGCTGACGAATGTCTTG-3' and -5kbR: 5'-GGGCTCATTTCACTGGTTGT-3'. Relative binding was calculated as the percent of input immunoprecipitated with KLF4 antibody for the Cx26 promoter amplicon compared with the amplicon $5 \mathrm{~kb}$ proximal to the $\mathrm{Cx} 26$ gene.

Immunohistochemistry and Western blot analyses. Back skin samples from E18.5 embryos or newborns were frozen directly or fixed overnight and then 
embedded in paraffin wax. Frozen sections were hybridized with a CX26 rabbit polyclonal antibody (1:100; Zymed Laboratories Inc.) and an integrin $\alpha 6$ rat monoclonal antibody (1:400; Chemicon International). Fluorescent secondary antibodies Alexa Fluor 488 goat anti-rabbit (1:400) and 594 goat anti-rat (1:400) (Invitrogen Corp.) were used and slides mounted with a DAPI glycerol medium (Invitrogen Corp.). Fluorescence was visualized on Zeiss Axioplot microscope, and images were captured with a Coolsnap Photometrix camera. Paraffin sections were hybridized with an integrin $\alpha 6$ rat monoclonal antibody (1:400; Chemicon International) and a K6 rabbit polyclonal antibody (1:500; Covance Research Products). Paraffin sections were hybridized with a BrdU mouse monoclonal antibody (1:500; clone BRD.2; Labvision) following the manufacturer's suggestions ( $\mathrm{HCl}$ and trypsin treatments) with the MOM kit (Vector Laboratories). The number of positive cells in 250 consecutive basal cells was counted and averaged in 3 nonoverlapping skin sections. Frozen sections were hybridized with CD3, CD4, and CD8 antibodies as previously described (47). For Western blot analysis, newborn skin was snap-frozen in liquid nitrogen and pulverized. Protein concentration was determined using the Lowry method (DC protein assay; Bio-rad). Equivalent amounts of protein $(\sim 10 \mu \mathrm{g})$ were resolved via SDS-PAGE (4-12\% Bis-Tris gels; Invitrogen Corp.) and electrophoresed onto Nitrocellulose (Invitrogen Corp.). Equal transfer was assessed with Ponceau S staining (Sigma-Aldrich). The blots were incubated with monoclonal CX26 antibody (Zymed Laboratories Inc.), followed by HRP-conjugated secondary antibodies and detection with ECL reagents (Amersham Biosciences).

Barrier function assays. We performed dye penetration assay with $\mathrm{X}$-gal at $\mathrm{pH} 4.5$ for approximately 4 hours at $37^{\circ} \mathrm{C}$ as described previously (11). Tail tips and spleens were removed for genotyping by PCR and FISH, respectively. After staining, embryos were photographed under an MZFLIII dissecting scope (Leica Microsystems) using a digital Axiocam camera (Zeiss), and images were acquired with Openlab software (version 4.0.3; Improvision). Transepidermal water loss was measured in newborn mice with the Tewameter (Courage \& Khazaka Electronic).

Electron microscopy. Whole skin was removed immediately following euthanasia and fixed overnight at $4^{\circ} \mathrm{C}$ in $2 \%$ glutaraldehyde, $2 \%$ paraformaldehyde, $\mathrm{CaCl}_{2}(2 \mathrm{mM})$ in sodium cacodylate $(0.1 \mathrm{M}, \mathrm{pH} 7.3)$. Samples were post-fixed briefly with $0.5 \%$ osmium tetroxide at $4{ }^{\circ} \mathrm{C}$ followed by $2 \%$ aqueous uranyl acetate. Samples were dehydrated in ethanol and embedded in LX-112 resin (Ladd Research).

In vivo dye transfer. Immediately following euthanasia, a 5- $\mathrm{mm}$ incision was made on the backs of newborn mice. A PBS solution containing $1 \%$ neurobiotin (Vector Laboratories) and 1\% rhodamine-dextran (Invitrogen Corp.) was applied inside the wound and incubated at $37^{\circ} \mathrm{C}$ for 10 minutes. The wound area was excised and washed in PBS 3 times before fixing in $4 \%$ PFA and embedding in paraffin wax. The sections were deparaffinized and stained with fluorescein streptavidin (1:1,000; Vector Laboratories), mounted, and visualized as described above.

Wound healing studies. After back hair was shaved, 4 full-thickness skin biopsies of $5 \mathrm{~mm}$ diameter were created using dermal biopsy punch (Milt- enyi Biotec). The extent of wound closure was determined by measuring the visible scab area. After euthanasia of the mouse, the wound areas were removed, fixed, and embedded in paraffin for histology.

ATP release from keratinocyte. Primary keratinocytes were cultured under standard conditions and then switched at $30-40 \%$ confluence to $0.2 \mathrm{mM}$ calcium for 24-36 hours (63). The cells were washed with PBS (1 mM calcium chloride and $1 \mathrm{mM}$ magnesium chloride), followed by a quick wash with PBS (free of calcium and magnesium). To induce opening of hemichannels, $250 \mu \mathrm{l}$ of calcium- and magnesium-free PBS was added to each well and incubated at $37^{\circ} \mathrm{C}$ for 5 minutes. The supernatant was collected and immediately placed on ice. The ATP levels were determined using an ATP determination kit (Invitrogen Corp.). The ATP concentration was normalized for the number of cells in each well.

Intracellular calcium measurements. Primary keratinocytes were plated on top of coverslips and cultured in $1.2 \mathrm{mM}$ calcium for 24 hours. Cells were washed and then incubated in Krebs-Ringer buffer, supplemented with $2 \mathrm{~mm}$ fura-2/AM, at $37^{\circ} \mathrm{C}$ for 60 minutes. Coverslips were washed and mounted on an Axiovent 135 microscope (Zeiss) with an Attofluor Digital Fluorescence Microscopy System attached (Atto Instruments Inc.). Multiple single cells were examined simultaneously with alternating 340- and 380-nm light beams, and the intensity of light emission at $520 \mathrm{~nm}$ was measured.

Statistics. Two-tailed Student's $t$ test was used to compare the mean values between different groups. $P$ values less than 0.05 were considered statistically significant.

\section{Acknowledgments}

This work was supported by the National Human Genome Research Institute and National Eye Institute Intramural Programs. We would like to thank members of the Stanley group, especially Jonathan Vogel, Mark Udey, Jere Stern, and Gabriele Richards for insightful comments. We also thank Anne Bowcock for her critical evaluation of the manuscript. Allen Li and XiaoJing Wang provided advice on the immunohistochemical staining of lymphocytes. The NIH's veterinary pathologists provided very useful consultations and technical expertise in histologic diagnosis. Animal care was provided wonderfully by Bahafta Berhe, James Ofari, and Donny Johnson. The National Human Genome Research Institute microscopy core facility, headed by Amalia Dutra, performed the FISH analysis. Thanks go to Julia Fekecs for assistance with figure preparation.

Received for publication October 21, 2005, and accepted in revised form March 7, 2006.

Address correspondence to: Julia Segre, National Human Genome Research Institute, NIH, 49 Convent Drive, Room 4A26, Bethesda, Maryland 20892, USA. Phone: (301) 402-2314; Fax: (301) 4024929; E-mail: jsegre@nhgri.nih.gov.
1. Segre, J.A. 2006. Epidermal barrier formation and recovery in skin disorders. J. Clin. Invest. 116:1150-1158. doi:10.1172/JCI28521.

2. Elias, P.M. 2005. Stratum corneum defensive functions: an integrated view. J. Invest. Dermatol. 125:183-200.

3. Cartlidge, P. 2000. The epidermal barrier. Semin. Neonatol. 5:273-280.

4. Nickoloff, B.J., and Nestle, F.O. 2004. Recent insights into the immunopathogenesis of psoriasis provide new therapeutic opportunities. J. Clin. Invest. 113:1664-1675. doi:10.1172/JCI200422147.

5. Schon, M.P., and Boehncke, W.H. 2005. Psoriasis. N. Engl. J. Med. 352:1899-1912.
6. Capon, F., et al. 2001. Fine mapping of the PSORS4 psoriasis susceptibility region on chromosome 1q21. J. Invest. Dermatol. 116:728-730.

7. Ghadially, R., Reed, J.T., and Elias, P.M. 1996. Stratum corneum structure and function correlates with phenotype in psoriasis. J. Invest. Dermatol. 107:558-564.

8. Wood, L.C., Jackson, S.M., Elias, P.M., Grunfeld, C., and Feingold, K.R. 1992. Cutaneous barrier perturbation stimulates cytokine production in the epidermis of mice. J. Clin. Invest. 90:482-487.

9. Proksch, E., Feingold, K.R., Man, M.Q., and Elias, P.M. 1991. Barrier function regulates epidermal DNA synthesis. J. Clin. Invest. 87:1668-1673.
10. Segre, J.A., Bauer, C., and Fuchs, E. 1999. Klf4 is a transcription factor required for establishing the barrier function of the skin. Nat. Genet. 22:356-360.

11. Hardman, M.J., Sisi, P., Banbury, D.N., and Byrne, C. 1998. Patterned acquisition of skin barrier function during development. Development. 125:1541-1552.

12. Jaubert, J., Cheng, J., and Segre, J.A. 2003. Ectopic expression of kruppel like factor 4 (Klf4) accelerates formation of the epidermal permeability barrier. Development. 130:2767-2777.

13. Zhou, X., et al. 2003. Novel mechanisms of T-cell and dendritic cell activation revealed by profiling of psoriasis on the 63,100-element oligonucleotide array. Physiol. Genomics. 13:69-78. 
14. Rivas, M.V., et al. 1997. Identification of aberrantly regulated genes in diseased skin using the cDNA differential display technique. J. Invest. Dermatol. 108:188-194.

15. Kretz, M., Maass, K., and Willecke, K. 2004. Expression and function of connexins in the epidermis, analyzed with transgenic mouse mutants. Eur. J. Cell Biol. 83:647-654.

16. Stout, C., Goodenough, D.A., and Paul, D.L. 2004. Connexins: functions without junctions. Curr. Opin. Cell Biol. 16:507-512.

17. Choudhry, R., Pitts, J.D., and Hodgins, M.B. 1997. Changing patterns of gap junctional intercellular communication and connexin distribution in mouse epidermis and hair follicles during embryonic development. Dev. Dyn. 210:417-430.

18. Coutinho, P., Qiu, C., Frank, S., Tamber, K., and Becker, D. 2003. Dynamic changes in connexin expression correlate with key events in the wound healing process. Cell Biol. Int. 27:525-541.

19. Goliger, J.A., and Paul, D.L. 1995. Wounding alters epidermal connexin expression and gap junctionmediated intercellular communication. Mol. Biol. Cell. 6:1491-1501.

20. Pedersen, T.X., et al. 2003. Laser capture microdissection-based in vivo genomic profiling of wound keratinocytes identifies similarities and differences to squamous cell carcinoma. Oncogene. 22:3964-3976.

21. Labarthe, M.P., Bosco, D., Saurat, J.H., Meda, P., and Salomon, D. 1998. Upregulation of connexin 26 between keratinocytes of psoriatic lesions. J. Invest. Dermatol. 111:72-76.

22. Lucke, T., et al. 1999. Upregulation of connexin 26 is a feature of keratinocyte differentiation in hyperproliferative epidermis, vaginal epithelium, and buccal epithelium. J. Invest. Dermatol. 112:354-361.

23. Kelsell, D.P. 2004. Connexin mutations in human disease. Exp. Dermatol. 13:661-662.

24. Richard, G. 2005. Connexin disorders of the skin. Clin. Dermatol. 23:23-32.

25. Iizuka, H., Takahashi, H., Honma, M., and IshidaYamamoto, A. 2004. Unique keratinization process in psoriasis: late differentiation markers are abolished because of the premature cell death. J. Dermatol. 31:271-276.

26. Martin, N., Patel, S., and Segre, J.A. 2004. Longrange comparison of human and mouse Sprr loci to identify conserved noncoding sequences involved in co-ordinate regulation. Genome Res. 14:2430-2438.

27. Patel, S., Kartasova, T., and Segre, J.A. 2003. Mouse Sprr locus: a tandem array of coordinately regulated genes. Mamm. Genome. 14:140-148.

28. Koch, P.J., et al. 2000. Lessons from loricrin-deficient mice: compensatory mechanisms maintaining skin barrier function in the absence of a major cornified envelope protein. J. Cell Biol. 151:389-400.

29. Wakabayashi, N., et al. 2003. Keap1-null mutation leads to postnatal lethality due to constitutive Nrf2 activation. Nat. Genet. 35:238-245.

30. Cook, P.W., Brown, J.R., Cornell, K.A., and Pittelkow, M.R. 2004. Suprabasal expression of human amphiregulin in the epidermis of transgenic mice induces a severe, early-onset, psoriasis-like skin pathology: expression of amphiregulin in the basal epidermis is also associated with synovitis. Exp. Dermatol. 13:347-356.

31. Cook, P.W., et al. 1997. Transgenic expression of the human amphiregulin gene induces a psoriasislike phenotype. J. Clin. Invest. 100:2286-2294.

32. Maass, K., et al. 2004. Defective epidermal barrier in neonatal mice lacking the C-terminal region of connexin43. Mol. Biol. Cell. 15:4597-4608.

33. Li, E.R., Owens, D.M., Djian, P., and Watt, F.M. 2000. Expression of involucrin in normal, hyperproliferative and neoplastic mouse keratinocytes. Exp. Dermatol. 9:431-438.

34. Eckert, R.L., et al. 2004. Regulation of involucrin gene expression. J. Invest. Dermatol. 123:13-22.

35. Jaubert, J., Patel, S., Cheng, J., and Segre, J.A. 2004. Tetracycline-regulated transactivators driven by the involucrin promoter to achieve epidermal conditional gene expression. J. Invest. Dermatol. 123:313-318.

36. Furuse, M., et al. 2002. Claudin-based tight junctions are crucial for the mammalian epidermal barrier: a lesson from claudin-1-deficient mice. J. Cell Biol. 156:1099-1111.

37. Tran Van Nhieu, G., et al. 2003. Connexin-dependent inter-cellular communication increases invasion and dissemination of Shigella in epithelial cells. Nat. Cell Biol. 5:720-726.

38. Rowland, B.D., Bernards, R., and Peeper, D.S. 2005. The KLF4 tumour suppressor is a transcriptional repressor of $\mathrm{p} 53$ that acts as a context-dependent oncogene. Nat. Cell Biol. 7:1074-1082.

39. McDonald, O.G., Wamhoff, B.R., Hoofnagle, M.H., and Owens, G.K. 2006. Control of SRF binding to CArG box chromatin regulates smooth muscle gene expression in vivo. J. Clin. Invest. 116:36-48. doi:10.1172/JCI26505.

40. Sano, S., et al. 2005. Stat 3 links activated keratinocytes and immunocytes required for development of psoriasis in a novel transgenic mouse model. Nat. Med. 11:43-49.

41. Georgiou, J.G., et al. 2005. Human epidermal and monocyte-derived langerhans cells express functional P2X receptors. J. Invest. Dermatol. 125:482-490.

42. Inoue, K., Denda, M., Tozaki, H., Fujishita, K., and Koizumi, S. 2005. Characterization of multiple $\mathrm{P} 2 \mathrm{X}$ receptors in cultured normal human epidermal keratinocytes. J. Invest. Dermatol. 124:756-763.

43. Pillai, S., and Bikle, D.D. 1992. Adenosine triphosphate stimulates phosphoinositide metabolism, mobilizes intracellular calcium, and inhibits terminal differentiation of human epidermal keratinocytes. J. Clin. Invest. 90:42-51.

44. Denda, M., Inoue, K., Fuziwara, S., and Denda, S. 2002. P2X purinergic receptor antagonist accelerates skin barrier repair and prevents epidermal hyperplasia induced by skin barrier disruption. J. Invest. Dermatol. 119:1034-1040.

45. Mizumoto, N., et al. 2002. CD39 is the dominant Langerhans cell-associated ecto-NTPDase: modulatory roles in inflammation and immune responsiveness. Nat. Med. 8:358-365.

46. Granstein, R.D., et al. 2005. Augmentation of cuta- neous immune re-sponses by ATPgammaS: purinergic agonists define a novel class of immunologic adjuvants. J. Immunol. 174:7725-7731.

47. Jan, A.Y., Amin, S., Ratajczak, P., Richard, G., and Sybert, V.P. 2004. Genetic heterogeneity of KID syndrome: identification of a Cx30 gene (GJB6) mutation in a patient with KID syndrome and congenital atrichia. J. Invest. Dermatol. 122:1108-1113.

48. Van Steensel, M.A., et al. 2004. A phenotype resembling the Clouston syndrome with deafness is associated with a novel missense GJB2 mutation. J. Invest. Dermatol. 123:291-293.

49. Essenfelder, G.M., et al. 2004. Connexin 30 mutations responsible for hidrotic ectodermal dysplasia cause abnormal hemichannel activity. Hum. Mol. Genet. 13:1703-1714.

50. Li, A.G., Wang, D., Feng, X.H., and Wang, X.J. 2004. Latent TGFbeta1 overexpression in keratinocytes results in a severe psoriasis-like skin disorder. EMBO J. 23:1770-1781.

51. Zenz, R., et al. 2005. Psoriasis-like skin disease and arthritis caused by inducible epidermal deletion of Jun proteins. Nature. 437:369-375.

52. Soumelis, V., et al. 2002. Human epithelial cells trigger dendritic cell mediated allergic inflammation by producing TSLP. Nat. Immunol. 3:673-680.

53. Zhou, B., et al. 2005. Thymic stromal lymphopoietin as a key initiator of allergic airway inflammation in mice. Nat. Immunol. 6:1047-1053.

54. Yoo, J., et al. 2005. Spontaneous atopic dermatitis in mice expressing an inducible thymic stromal lymphopoietin transgene specifically in the skin. J. Exp. Med. 202:541-549.

55. Al-Shami, A., Spolski, R., Kelly, J., Keane-Myers, A., and Leonard, W.J. 2005. A role for TSLP in the development of inflammation in an asthma model. J. Exp. Med. 202:829-839.

56. Chavanas, S., et al. 2000. Mutations in SPINK5, encoding a serine protease inhibitor, cause Netherton syndrome. Nat. Genet. 25:141-142.

57. Walley, A.J., et al. 2001. Gene polymorphism in Netherton and common atopic disease. Nat. Genet. 29:175-178.

58. Cookson, W.O., et al. 2001. Genetic linkage of childhood atopic dermatitis to psoriasis susceptibility loci. Nat. Genet. 27:372-373.

59. Aszterbaum, M., Feingold, K.R., Menon, G.K., and Williams, M.L. 1993. Glucocorticoids accelerate fetal maturation of the epidermal permeability barrier in the rat. J. Clin. Invest. 91:2703-2708.

60. Kretz, M., et al. 2003. Altered connexin expression and wound healing in the epidermis of connexindeficient mice. J. Cell Sci. 116:3443-3452.

61. Qiu, C., et al. 2003. Targeting connexin 43 expression accelerates the rate of wound repair. Curr. Biol. 13:1697-1703.

62. Romano, R.A., Birkaya, B., and Sinha, S. 2006. Defining the regulatory elements in the proximal promoter of DNp63 in Keratinocytes: potential roles for $\mathrm{Sp} 1 / \mathrm{Sp} 3, \mathrm{NF}-\mathrm{Y}$ and $\mathrm{p} 63$. J. Invest. Dermatol. In press.

63. Hennings, H., et al. 1980. Calcium regulation of growth and differentiation of mouse epidermal cells in culture. Cell. 19:245-254. 